\title{
Penile Reconstruction with Skin Grafts and Dermal Matrices: Indications and Management
}

\author{
Paloma Triana Junco ${ }^{1}$ Mariela Dore ${ }^{1}$ Vanesa Nuñez Cerezo ${ }^{2}$ Javier Jimenez Gomez ${ }^{1}$ \\ Miriam Miguel Ferrero ${ }^{2}$ Mercedes Díaz González ${ }^{2}$ Pedro Lopez-Pereira ${ }^{3}$ Juan Carlos Lopez-Gutierrez ${ }^{4}$
}

${ }^{1}$ Department of Pediatric Surgery, Hospital Universitario La Paz, Madrid, Spain

2 Department of Pediatric Surgery, Hospital La Paz, Madrid, Spain

${ }^{3}$ Department of Pediatric Urology, Hospital Universitario La Paz, Madrid, Spain

${ }^{4}$ Department of Pediatric Surgery, Division of Vascular Anomalies, La Paz Children's Hospital, Madrid, Spain

Eur J Pediatr Surg Rep 2017;5:e47-e50.
Address for correspondence Paloma Triana Junco, MD, Department of Pediatric Surgery, Hospital Universitario La Paz, Paseo de la Castellana 261, Madrid 28046, Spain (e-mail: pa_triana@hotmail.com).

\section{Abstract \\ Keywords \\ - penile reconstruction \\ - skin grafts \\ - dermal matrices \\ - pediatric \\ Introduction The penis eventually needs specific cutaneous coverage in the context of reconstructive procedures following trauma or congenital anomalies. Local flaps are the first choice but are not always available after multiple previous procedures. In these cases, skin graft and dermal matrices should be considered. \\ Materials and Methods This study was a retrospective review of the past 4 years of four patients with severe loss of penile shaft skin who underwent skin reconstruction. Dermal matrices and skin grafts were utilized. Dermal matrices were placed for a median of 4.5 weeks (3.0-6.0 weeks). The skin graft was harvested from the inner thigh region for split-thickness skin graft (STSG) and the inguinal region for full- thickness skin graft (FTSG). \\ Results The four patients presented with complete loss of skin in the penile shaft. One patient had a vesical exstrophy, one had a buried penis with only one corpus cavernosum, one had a wide congenital lymphedema of the genitalia, and one had a lack of skin following circumcision at home. They underwent reconstruction with three patients undergoing split-thickness skin graft; two dermal matrices; and one full- thickness graft, respectively, thereby achieving a good cosmetic and functional result. There were no complications, and all the patients successfully accepted the graft. Conclusion Dermal matrices and skin grafts may serve as effective tools in the management of severe penile skin defects unable to be covered with local flaps.}

\section{New Insights and the Importance for the Pediatric Surgeon}

Dermal matrices and skin grafts can be an effective tool to provide cutaneous coverage in the management of severe penile skin defects unable to be covered with local flaps.

received

June 11, 2017

accepted after revision

July 16, 2017
DOI https://doi.org/

10.1055/s-0037-1606282.

ISSN 2194-7619. (c) 2017 Georg Thieme Verlag KG

Stuttgart · New York
License terms

(c) (1) 


\section{Introduction}

The penis eventually needs specific cutaneous coverage in the context of reconstructive procedures following trauma or congenital anomalies. The preferred choice of pediatric urologists is the use of excess preputial skin rotating flaps from redundant areas such as scrotum. ${ }^{1}$ However, local flaps are not always available, mainly after multiple previous surgical procedures. Additional options for cutaneous coverage include the use of dermal matrices, full-thickness skin grafts (FTSGs), and split-thickness skin grafts (STSGs). ${ }^{2}$ Selection of the technique depends on the size and location of the defect and is subjected to the vascular compromises usually associated with skin grafts. Since penile size changes with erection, FTSG may be preferred for its greater elasticity and less primary contraction after harvesting. But STSG requires less ideal conditions for survival and have lower incidence of graft failure. $^{2}$

We here present our series of children with severe penile shaft skin loss who were treated with dermal matrices and skin grafts in the past 4 years.

\section{Materials and Methods}

We performed a retrospective review of four patients who underwent skin reconstruction due to severe loss of penile shaft skin in the past 4 years (2012-2016).

We collected data on demographic characteristics of patients, cause of penile defect, location and wideness of the lesions, associated complications, types of cutaneous coverage, length of treatment, and results.

Patients were treated with both dermal matrices and skin grafts. After debridement, dermal matrices (Integra) were attached with staples and kept in place for a mean of 4.5 weeks (3.0-6.0 weeks) covered with antimicrobial silver dressings (Acticoat) with weekly changes. Split-thickness skin grafts (STSGs) were harvested from the inner thigh with an electric dermatome, and fenestration was performed with the "piecrusted" method. Full-thickness skin grafts (FTSGs) were harvested from the inguinal region with a surgical knife.
Both types of skin grafts were attached with interrupted absorbable sutures and covered with "tie-over" bolster dressings maintained for 7 days before uncovering the graft. Donor sites were covered with occlusive dressings with changes every 24 or 48 hours until complete healing. Patients were followed up after surgery weekly for the first month at the outpatient clinic and monthly for the first 3 months.

A descriptive analysis was performed. Data were expressed in percentage from total and medians with their ranks.

\section{Results}

\section{Characteristics of Patients}

All four patients were males with a mean age of 10 years (4-14) at the time of reconstruction. One patient presented with complete loss of the skin in the penile shaft following ritual circumcision at home, second due to wide congenital lymphedema, third after previous multiple surgeries in the context of vesical exstrophy, and fourth due to a buried penis with only one corpus cavernosum (-Figs. 1-4).

\section{Treatment}

Under the combination of general and epidural anesthesia, a urethral catheter was inserted and full degloving of penile shaft was performed. Artificial erection test was needed in one patient. Two patients underwent reconstruction with dermal matrices (Integra) following STSG, one was covered only with STSG, and last one received FTSG (-Figs. 1-4).

\section{Efficacy and Safety}

Successful acceptance of the graft was achieved in $100 \%$ of patients, with patients and parents reported good cosmetic and functional results. There were no complications. The mean follow-up was 2 years (1-4 years).

\section{Discussion}

Using dermal matrices and skin grafts for reconstruction in penile skin loss remains a valuable option when there is not enough local skin for coverage, usually due to prior surgical
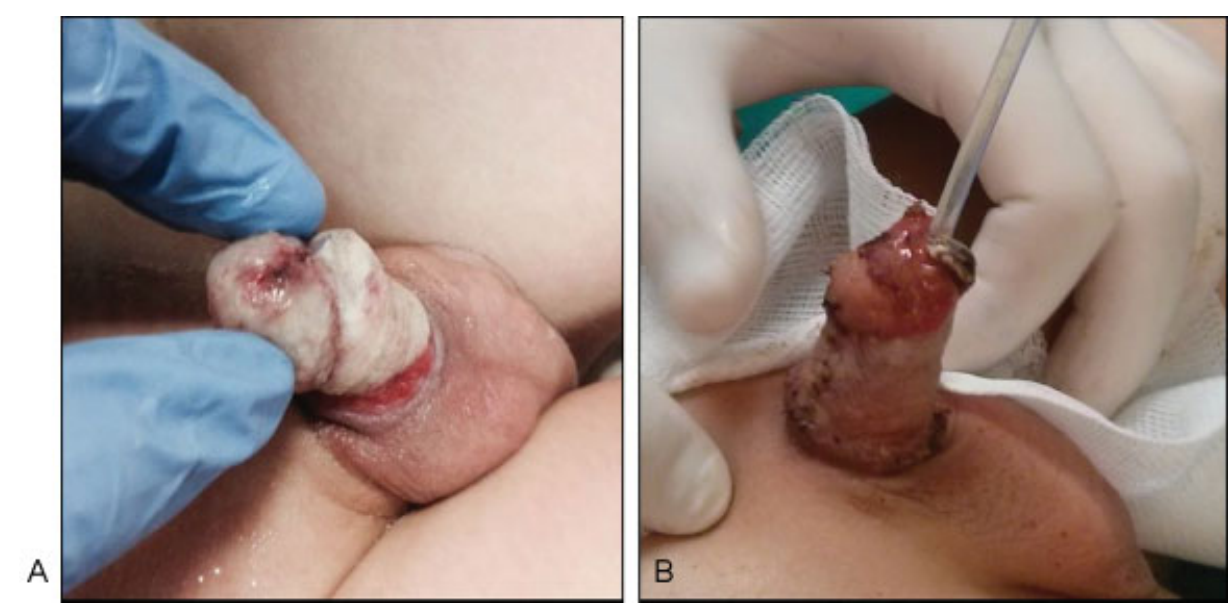

Fig. 1 (A) Lack of penile skin after ritual circumcision. (B) Reconstruction with FTSG 1 month after circumcision, at 4 years of age. FTSG, fullthickness skin graft. 

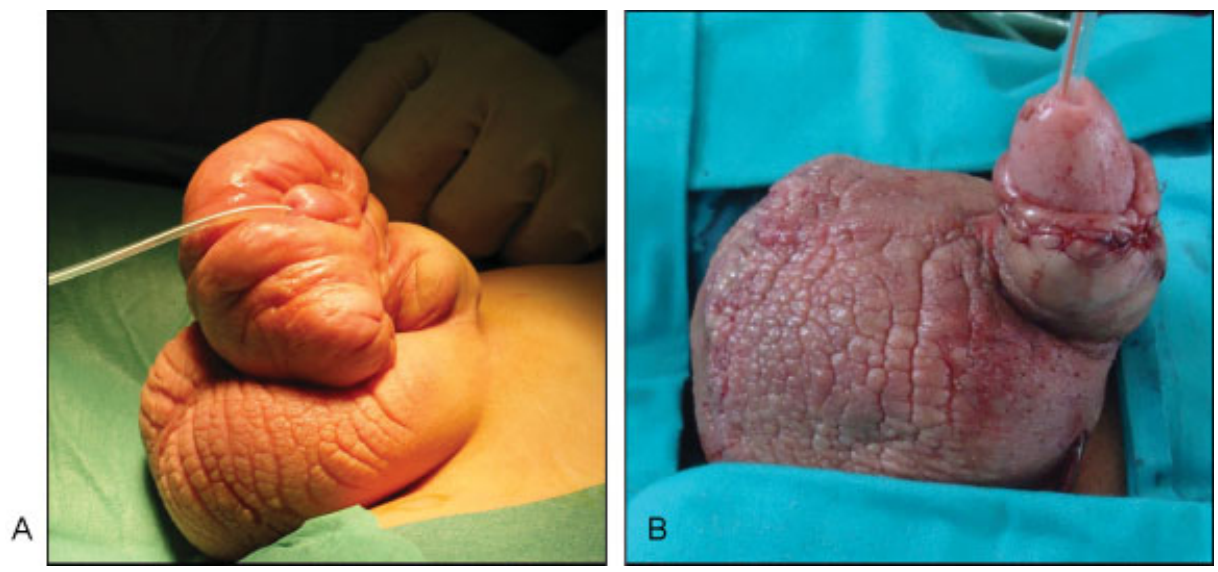

Fig. 2 (A) Congenital lymphedema of the penis. (B) Reconstruction with STSG at 7 years of age. STSG, split-thickness skin graft.

A

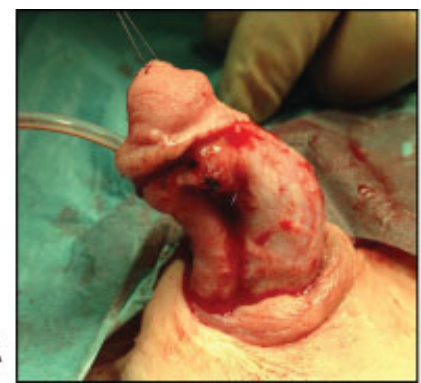

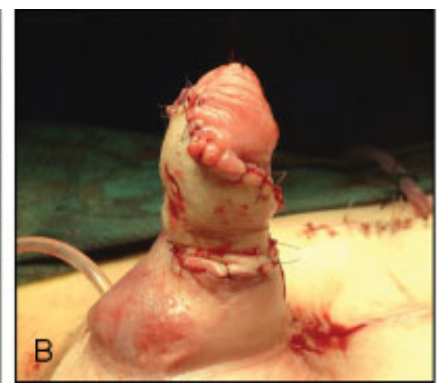

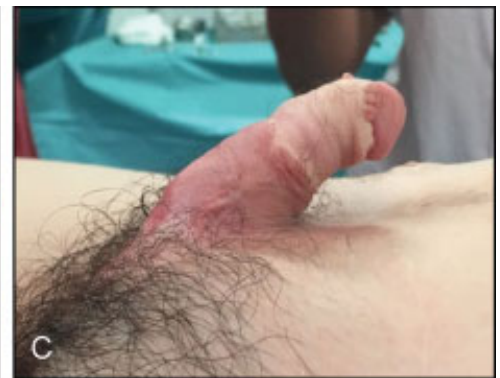

Fig. 3 (A) Lack of penile skin after multiple previous surgeries in a vesical exstrophy patient. (B) Reconstruction with dermal matrix and STSG at 12 years of age. (C) Reconstructed penis 1 month after surgery. STSG, split-thickness skin graft.

A

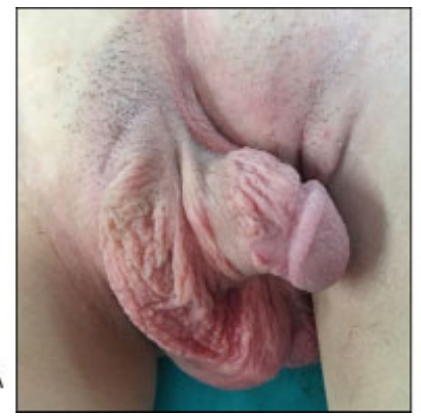

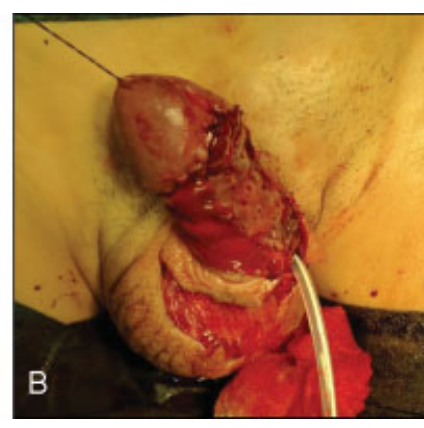

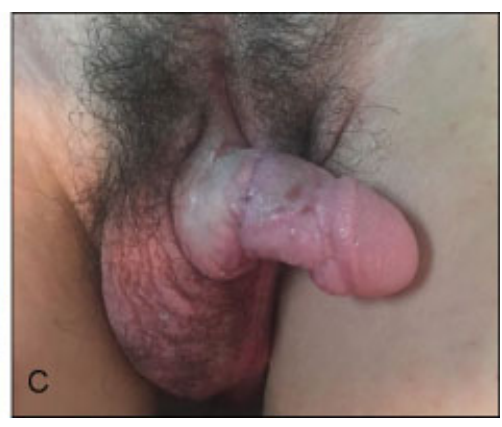

Fig. 4 (A) Lack of penile skin and severe chordee due to a buried penis with only one corpus cavernosum. (B) Reconstruction with dermal matrix and STSG at 14 years of age. (C) Reconstructed penis 1 month after surgery. STSG, split-thickness skin graft.

interventions. There is still an ongoing debate over which type of skin graft should be used to replace the penile skin defect. Sir Harold Gilles suggested in 1917 the core principle of reconstructive surgery: "tissue loss is replacing like with like," meaning donor tissue should be the one that most closely replicates the native tissue in function and cosmetic appearance. $^{3}$ FTSGs show significantly more primary contraction than STSGs, but STSGs present lower incidence of graft failure and have much broader range of application than FTSGs. ${ }^{2}$ It should also be noted that during penile reconstructive surgery, the penis should be at full erection, when the graft and dressing are applied, to prevent wrinkling and contracture of the graft, which would lead to rejection of graft. All our patients reported good cosmetic and functional results without any shrinkage of the graft or subsequent curvature or chordee. Parents reported suitable erections although patients were not sexually active; so, they would need further follow-up.

While choosing a skin graft, donor-site morbidity must also be considered. A FTSG leaves a full-thickness defect at the donor site and large donor sites may be difficult to close or hide, while STSG usually leaves a superficial wound that heals easily. Location of the donor site is also important, i.e., choosing areas that are usually covered by clothes and are easy to procure a wide skin graft, such as medial or posterior thigh for STSG and inguinal region for FTSG. ${ }^{2}$ All our patients achieved good cosmetic results from their donor sites. 
Whereas skin grafting has been widely used and published for penile reconstruction, ${ }^{4-9}$ dermal matrices have not yet played a leading role for these defects. Dermal matrices have been used in the past for penile augmentation. ${ }^{10}$ These have been rarely employed for penile skin defects and were only utilized in adults with Fournier's gangrene before STSG. ${ }^{11,12}$ Our two patients treated with dermal matrices before STSG had longer treatment duration but exhibited same cosmetic and functional results. Therefore, dermal matrices remain a good option for penile defect coverage.

\section{Conclusion}

Dermal matrices and skin grafts may serve as effective tools to provide cutaneous coverage in the management of severe penile skin defects unable to be covered with local flaps. Patients achieved a good cosmetic and functional result with no differences between the performed techniques.

\section{Conflict of Interest}

None.

\section{References}

1 Paediatric Urology Web book. 2nd ed. European Society for Paediatric Urology; 2014

2 White N, Hettiaratchy S, Papini RP. The choice of split-thickness skin graft donor site: patients' and surgeons' preferences. Plast Reconstr Surg 2003;112(03):933-934
3 Thakar HJ, Dugi DD III. Skin grafting of the penis. Urol Clin North Am 2013;40(03):439-448

4 Alwaal A, McAninch JW, Harris CR, Breyer BN. Utilities of splitthickness skin grafting for male genital reconstruction. Urology 2015;86(04):835-839

5 Thompson JH, Zmaj P, Cummings JM, Steinhardt GF. An approach for using full thickness skin grafts for complex penile surgeries in children. J Urol 2006;175(05):1869-1871; discussion 1871

6 Asimakopoulos AD, Iorio B, Vespasiani G, Cervelli V, Spera E. Autologous split-thickness skin graft for penile coverage in the treatment of buried (trapped) penis after radical circumcision. BJU Int 2012;110(04):602-606

7 Gillett MD, Rathbun SR, Husmann DA, Clay RP, Kramer SA. Splitthickness skin graft for the management of concealed penis. J Urol 2005;173(02):579-582

8 Chertin B, Kocherov S, Binenboym R, et al. Fenestrated sheet splitthickness skin grafting for reconstruction of penile skin loss in pediatric population. J Pediatr Surg 2016;51(08):1362-1365

9 Díaz EC, Corcoran JF, Johnson EK. Pediatric penile reconstruction using autologous split-thickness skin graft. J Pediatr Urol 2016;12 (03):185-186

10 Alei G, Letizia P, Ricottilli F, et al. Original technique for penile girth augmentation through porcine dermal acellular grafts: results in a 69-patient series. J Sex Med 2012;9(07): 1945-1953

11 Ludolph I, Titel T, Beier JP, et al. Penile reconstruction with dermal template and vacuum therapy in severe skin and soft tissue defects caused by Fournier's gangrene and hidradenitis suppurativa. Int Wound J 2016;13(01):77-81

12 Zhang Z, Lv L, Mamat M, et al. Xenogenic (porcine) acellular dermal matrix promotes growth of granulation tissues in the wound healing of Fournier gangrene. Am Surg 2015;81(01): 92-95 